\title{
Sensors Based on Monochromatic Interrogation of a Localised Surface Plasmon Resonance
}

\author{
Catherine S. Kealley, Matthew D. Arnold, Alexander Porkovich, Michael B. Cortie* \\ Institute for Nanoscale Technology, University of Technology Sydney, PO Box 123, Broadway, \\ NSW 2007, Australia. \\ *E-mail: michael.cortie@uts.edu.au; Tel: +61-2-9514-2208 ; Fax: +61-2-9514-8340.
}

\begin{abstract}
The localised surface plasmon resonance in gold nanoparticles can be used as the basis of a refractometric sensor. Usually, this is accomplished by monitoring a shift in wavelength of the resonance peak, a task which requires measurements over a range of wavelengths. Here we investigate a different scheme, in which interrogation of the sensor is carried out at a single wavelength. We have used numerical simulations to estimate the effect that the shape of gold nanoparticles would have on the performance on such sensors. A variety of geometries of gold nanoparticles were investigated, including nano-spheres, nano-rods, nano-triangles, and nanobowties. The performance of a sensor that operates at a single wavelength is controlled by $\mathrm{d} T / \mathrm{d} n$, the change in transmittance $T$ with refractive index $n$, determined at the interrogation wavelength. In turn, $\mathrm{d} T / \mathrm{d} n$ depends upon the extinction cross-section of the nanoparticles at the chosen wavelength, and on the density of the nanoparticles in the light path. Contributions to the sensor efficiency also include the shift in wavelength of the plasmon resonance and, importantly, the peak sharpness. Of the particles examined, gold nanorods will provide the most sensitive sensors by a large margin.
\end{abstract}

Keywords: Refractometric sensor, Plasmon resonance, Gold nanoparticles, Particle shape, Sensor efficiency.

\section{Introduction}

The electrochemical, optical, chemical and physical properties of nanomaterials are strongly dependent on surface phenomena as a result of their very high ratio of surface-to-volume [1]. This makes nanomaterials useful in diverse sensor applications [2, 3]. Gold nanoparticles (GNPs) are particularly useful in these contexts as the result of their possessing several unusual attributes. Due to their particular surface chemistry, GNPs are useful scaffolds on which to assemble organic molecules, including proteins or fluorescent moieties. They are otherwise, however, conveniently inert in most analytes of interest. Gold nanoparticles are readily synthesised and may also be deposited onto substrates in regular arrays or with controlled morphologies using a wide variety of techniques [4] and they are electrical conductors. Finally, GNPs undergo a 
resonance with light, which causes them to have optical properties in the visible and nearinfrared parts of the spectrum that are quite unlike those of bulk gold [5]. The interaction of light with the free electrons in the metal nanoparticle gives rise to a localised surface plasmon resonance (LSPR), a collective oscillation of the charge density at optical frequencies. The wavelength at which these resonances peak is sensitive to the refractive index of the surrounding medium and to the morphology and size of the nanoparticles themselves. Therefore, gold nanoparticles can be used as the basis of a refractometric sensing technology. Other transduction possibilities also exist, for example, gold nanoparticles can be used to produce a surface enhanced Raman signal, they can enhance the fluorescence of nearby molecules, or they can be exploited as chemiresistors [3]. However, we will only consider refractometric sensors based on LSPRs here.

\subsection{Background: Refractometric Sensors Based on LSPR}

It is important to differentiate refractometric sensors based on LSPR from those based on surface plasmon polaritons (SPPs) propagating along the surface of semi-infinite gold films. Actually, sensors of this latter type are now well established and are widely used for biological determinations, particularly for the characterisation and quantification of binding events [6, 7]. These devices generally operate in total internal reflection mode using the Kretschmann configuration [6]. Confusingly, they are often referred to as surface plasmon resonance (SPR) sensors although, according to modern terminology, e.g.[8], they are actually based on SPPs. We will not consider this latter type of sensor here.

The LSPR sensor is a highly specialised optical technique for determining refractive index changes occurring within the optical near-field of a nanoparticle (the 'near-field' is that region of medium within several nanometers of a nanoparticle surface). The selective absorption and scattering of particular wavelengths of light will produce sensible colours, and colour changes, if the phenomena occur in the visible part of the spectrum. These phenomena are potentially useful for a variety of applications, such as chemical and biomolecular sensing, nanoscale optical components or devices, and surface-enhanced spectroscopies [2, 9]. The LSPR technique offers a unique opportunity to observe surface phenomena and molecular binding of species in real time [2]. The exact position, shape and intensity of the localised nanoparticle SPR can be controlled through factors such as the particle's morphology (size and shape) and dielectric environment (coating, surrounding medium, supporting substrate) [3]. Furthermore, the SPR characteristics can be readily accessed by relatively simple spectral interrogation in a transmission configuration. It is this versatility that is the basis for the current interest in LSPR sensors.

Most work in this field has exploited the change in peak position of the LSPR, induced by some localised change in refractive index. A paper by Himmelhaus and Takei in 2000, in which gold semi-shells are used to detect the binding of organic molecules is an early example of such a sensor, however, since then the group of Van Duyne and Schatz at Northwestern University in the USA has been particularly productive in this area. The reader is referred to various reviews for a discussion of progress up to end of 2006 [10-13]. A transmission geometry has been 
generally adopted for such LSPR-based sensors, e.g. sensors based on gold [14] or silver nanoparticles [15] immobilised on glass. However, a few investigators have considered the peak shift in the scattered spectrum e.g. (McFarland 2003) while others, eg. Brolo et al., have demonstrated that a periodic array of sub-wavelength holes on gold films can be utilised as a sensor [16]. A computational and experimental study to determine the variation of plasmon wavelength with size, shape and dielectric environment of anisotropic noble metal (gold and silver) nanoparticles was undertaken by Hao et al. [17]. Silver nanodisks and gold 'multipods' were identified as being promising. Lee and El-Sayed [18] and Chen et al. [19] identified Au or Ag nano-rods as being very suitable for these applications, and investigated the dependence of the sensitivity of their surface plasmon resonance (frequency and bandwidth) to changes in the surrounding environment, the size and shape of nano-rods and their ratio of $\mathrm{Au}$ to $\mathrm{Ag}$. They predicted that the spectral sensitivity of refractometric sensing systems based on these nano-rods would be increased when the plasmon resonance is shifted to lower energies by an increase in the aspect ratio. Rods with large aspect ratios provided better index sensitivity but nano-bipyramids yielded a superior figure-of-merit (obtained by dividing the shift in peak position by the peak width at half maximum) [20]. Finally, Nusz et al. have developed an analytical model that optimises the geometry of the gold nano-rod, and can be used for the rational design of a biosensor based on shifts in the SPR of individual Au nanoparticles [21].

It is clear that a large variety of nanoparticle shapes have been assessed for these 'peak position' sensors. Their sensitivity, defined as the shift in resonance peak per refractive index unit (RIU), varies from 40 to nearly $900 \mathrm{~nm} \mathrm{RIU}^{-1}$, with the lower figure corresponding to nano-spheres, and the higher figure corresponding to complex shapes such as multiply-branched nano-rods ('multipods'), nano-crescents or nanorice [9, 20, 22]. Sensitivities of about $400 \mathrm{~nm}$ $\mathrm{RIU}^{-1}$ have been reported for LSPR schemes based on nano-holes in a gold film [16] and for ordinary Au nano-rods [18]. Note, however, that an alternative sensitivity expressed in eV.RIU ${ }^{-1}$ shows the opposite trend, with the complex shapes ostensibly having lower sensitivity because their resonances are shifted to much lower photon energies [22]. Curiously, when this latter sensitivity is normalised against photon energy of the resonance peak most structures fall into a narrow band of 20 to $30 \% \mathrm{RIU}^{-1}$ anyway. Another, possibly more reliable, indicator of sensitivity is the sensing figure-of-merit proposed by Van Duyne and co-workers [23] obtained by normalising either nm.RIU ${ }^{-1}$ or eV.RIU ${ }^{-1}$ by the width of the resonance peak at half maximum.

Determination of the refractive index from position of the LSPR peak requires measurements to be made over a range of wavelengths. This increases the complexity and cost of the sensor system that would be required. In the present work we investigate whether a simpler technique, that of interrogating the sensor at a single, fixed, wavelength, is feasible, and if so, which nanoparticle shapes would be the best. This configuration eliminates the need to measure over a range of wavelengths but does introduce the requirement to have a reference beam for calibration of the relative signal intensity. 


\section{Methodology}

\subsection{Prediction of optical properties}

The optical responses of four different nanoparticle shapes have been simulated. These were: (1) nano-spheres of $40 \mathrm{~nm}$ diameter, (2) nano-rods (rod length $80 \mathrm{~nm}$, rod diameter $20 \mathrm{~nm}$ ), (3) nano-triangles (side length $40 \mathrm{~nm}$, height $10 \mathrm{~nm}$ ), and (4) nano-bowties (side length $40 \mathrm{~nm}$, height $10 \mathrm{~nm}$ ). Each optical response was calculated for five refractive indices; $n=1.33,1.37$, 1.40, 1.45 and 1.50 (note: $k=0$ in these simulations). The optical extinction characteristics of the simulated structures were calculated by a method based on the discrete dipole approximation code of Draine and Flatau [24, 25] In this scheme the nanostructure is approximated by a threedimensional array of polarisable dipoles. The optical response of this array to an incident electromagnetic field is then calculated. The applicability and accuracy of this technique has been previously verified by various investigators [5, 26, 27], and its particular advantage is that it can be applied to arbitrarily-shaped particles in an a priori fashion. The method is accurate provided the array of dipoles is sufficiently fine to provide a reasonable rendition of the geometry. We calculate only the results for gold because it will exhibit the greatest stability in a range of chemical environments.

Calculations were performed for the case of particles both with and without glass slabs, using the published complex refractive indices of gold and glass [28]. Arrays of at least 33000 dipoles per particle were applied in the calculations, sufficient to satisfy the DDSCAT code's requirements for accuracy and convergence. The calculated optical properties are expressed in terms of an extinction efficiency, $Q_{\text {ext }}$. In the case of the DDSCAT code, this is the ostensible optical extinction cross-section normalised by the nominal geometric cross-sectional area of the particle, expressed as if all the matter in the target was deformed into a sphere [25].

\subsection{Calculation of Sensor Efficiency}

Our analysis here is for the case where transduction is achieved by measurement of the intensity of light, $I_{t}$, transmitted through the sensor and falling on a detector. Furthermore, we assume that the detector output signal is linearly proportional to $I_{t}$.

The data reduction process used is illustrated in Figure 1, which is for the case of an LSPR sensor based on a colloidal suspension of simple gold nano-spheres. First the extinction efficiency, $Q_{\text {ext }}$, of the nanostructure is calculated for several different refractive indices spanning the range of interest (Figure 1(a)), then $Q_{\text {ext }}$ as a function of refractive index is determined for the range of wavelengths over which $Q_{\text {ext }}$ is more sensitive to $n$ (Figure 1(b)). In Figure 1(c) we have plotted $\mathrm{d} Q_{\mathrm{ext}} / \mathrm{d} n$ against $\lambda$, evaluated at a nominal refractive index of 1.400 . It is clear that the $\mathrm{d} Q_{\text {ext }} / \mathrm{d} n$ in this case peaks at an interrogation wavelength of close to $560 \mathrm{~nm}$. But is this the optimum wavelength at which to operate the sensor? It is actually not $Q_{\text {ext }}$ that is measured in the sensor but rather the transmittance of the incident beam, $T$. The actual sensitivity of the device at the chosen wavelength of measurement is given by

$$
S\left(\lambda, n_{0}\right)=\frac{\mathrm{d} T(\lambda)}{\mathrm{d} n}
$$


where $n$ is the variable refractive index of the analyte, and $S$ is the sensitivity of the sensor determined at wavelength $\lambda$ and a reference refractive index, $\mathrm{n}_{0}$. The optimum wavelength to operate at is the one that will give the maximum $S\left(\lambda, n_{0}\right)$. 


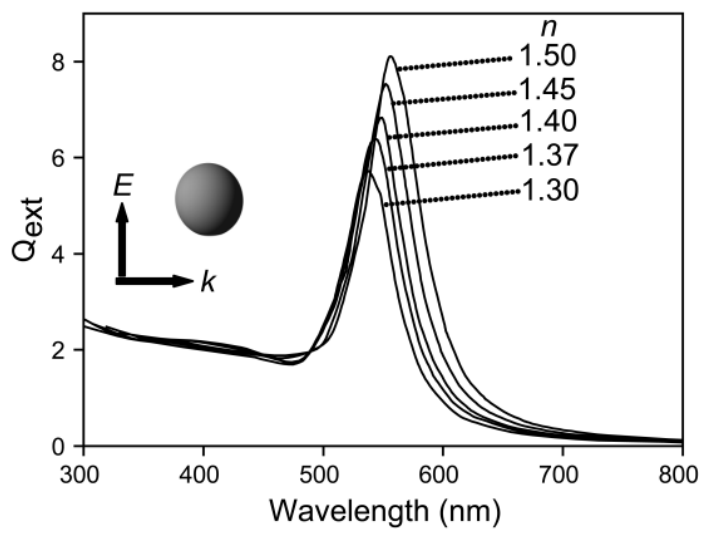

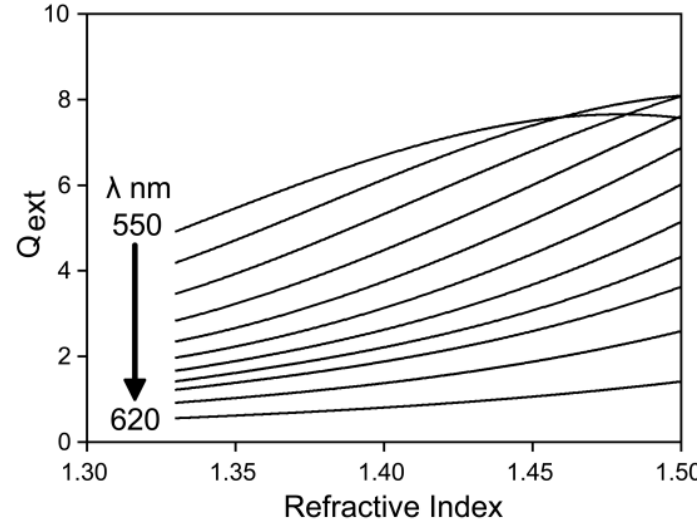

(b)

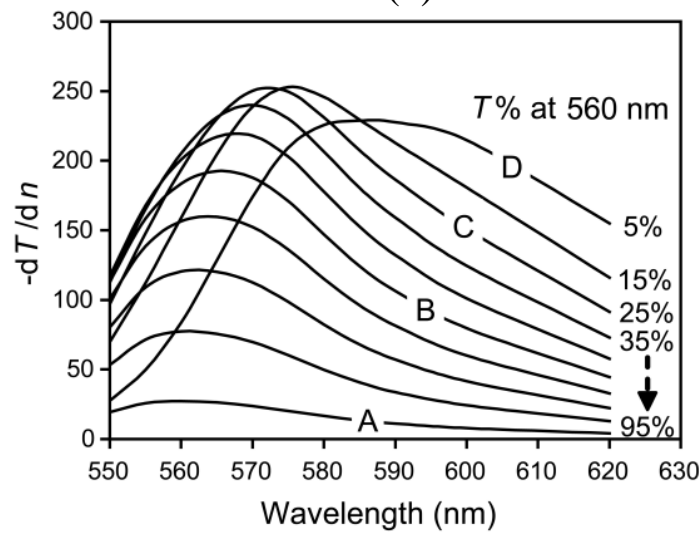

(d)

(a)

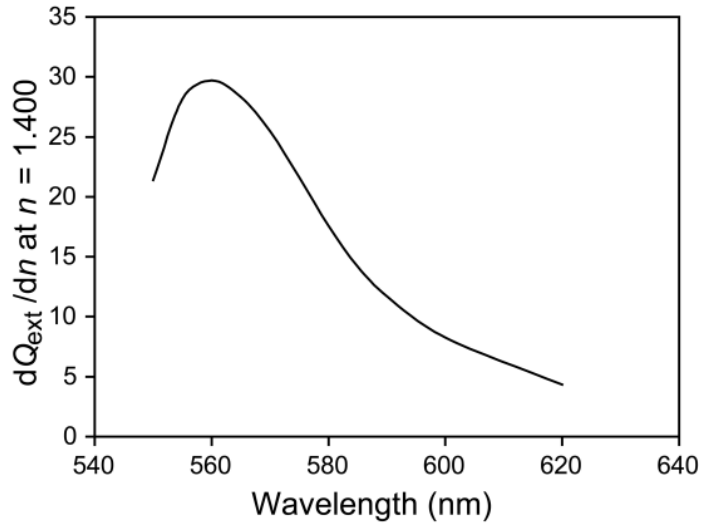

(c)

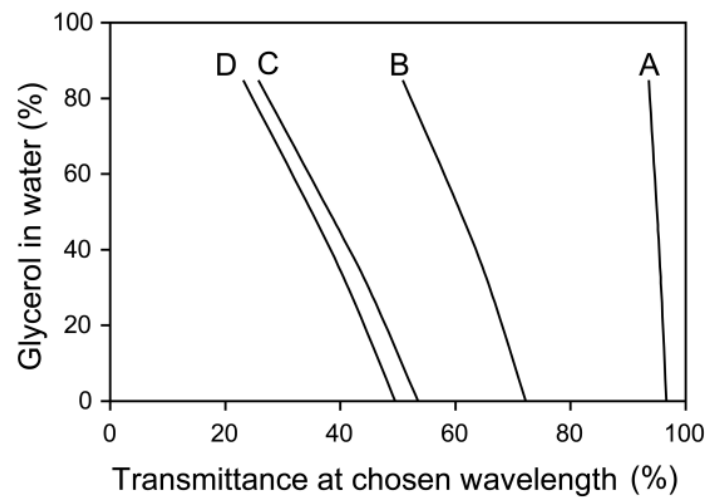

(e)

Figure 1 Method used to simulate the efficiency of the sensor, using the example of a colloidal suspension of gold nano-spheres. (a) Calculated extinction efficiency, $Q_{\text {ext }}, v s$ wavelength, $\lambda$, for an individual $52 \mathrm{~nm}$ diameter gold sphere immersed in media of the indicated refractive index. (b) $Q_{\text {ext }}$ vs $n$ curves determined for interrogation wavelengths between 550 and $620 \mathrm{~nm}$. (c) $\mathrm{d} Q_{\text {ext }} / \mathrm{d} n$ evaluated at a nominal $n$ of 1.400 , and plotted against interrogation wavelength. (d) $\mathrm{d} T / \mathrm{d} n$ plotted against both wavelength and optical thickness. The reference transmittances shown are taken at $560 \mathrm{~nm}$. Curves A to D are representative examples. (e) Application of curves A to D in a monochromatic LSPR sensor using the concentration of glycerol in water as an illustrative example. 
There are two extremes of geometry for these sensors. In the first, the optical path length through the sensing volume is significant, and the sensing nanoparticles are both dilute and randomly distributed throughout the volume of the optical path. In this case, the transmittance can be estimated (provided that multiple scattering events are negligible[29]) by the BeerLambert law:

$$
T=\frac{I_{t}}{I_{0}}=10^{-A}
$$

where $I_{o}$ is the intensity of the incident beam, $I_{\mathrm{t}}$ is the transmitted intensity and $A$ is the sample absorbance. We will designate this as the 'colloidal geometry'. The sample absorbance of the 'colloidal geometry' is related to the extinction cross-section of the individual nanoparticles by:

$$
A=\frac{N . x \cdot C_{\text {ext. }}}{2.303}
$$

where $N$ the number of particles per unit volume, $x$ the thickness of the sensor coating, and $C_{\text {ext }}$ the extinction cross-section[30]. The term N.x. $C_{\text {eff }}$ is a measure of the 'optical thickness' of the sample [29]. Since

$$
C_{\text {ext }}=\pi a_{e f f}^{2} \cdot Q_{e x t}
$$

where $a_{\text {eff }}$ is the 'effective radius' [25], it can be shown that

$$
T(\lambda, n)=e^{-\pi u_{\text {eff }}^{2} N x Q_{e x}(\lambda, n)}
$$

where both $T$ and $Q_{\text {ext }}$ depend on the wavelength of measurement and the refractive index of the medium. Clearly, $T$ depends on both $Q_{\text {ext }}$ and on the density of nanoparticles present in the coating $(N . x)$. As the concentration of the nanoparticles increases, Eq. (3) will start to underestimate the extinction[29] and hence over-estimate the transmittance. 
At any particular $\lambda$

$$
S=\frac{\mathrm{d} T}{\mathrm{~d} n}=-\pi a_{e f f}^{2} N x \cdot e^{-\pi a_{e f f}^{2} N x Q_{e x t}(\lambda, n)} \cdot \frac{\mathrm{d} Q_{e x t}}{\mathrm{~d} n}
$$

where the quantities are evaluated at the nominal refractive index of interest. The various parameters interact in a non-linear fashion because of the exponential relationship between $T$ and $A$, and hence, ultimately, between sensor output voltage and $Q_{\text {ext }}$.

In Figure $1(\mathrm{~d})$ we show $\mathrm{d} T / \mathrm{d} n$ evaluated at $n=1.400$, plotted against $\lambda$ for several optical thicknesses obtained by varying the density of the nanoparticles. The optical thicknesses were chosen to give transmittances ranging from 5\% to $95 \%$ at a reference wavelength of $560 \mathrm{~nm}$.

There is clearly a optimum region in which to operate the sensor. Specifically, in this case it is best to use coatings with transmittances that would be in the range of 15 to $25 \%$. However, the actual optimum wavelength at which to interrogate the sensor turns out to be between 575 and $580 \mathrm{~nm}$, considerably red-shifted compared to the position of peak $\mathrm{d} Q / \mathrm{d} n$ shown in Figure 1 (c) . Finally, $T$ can be related to the concentration of the particular analyte, for example glycerol in water (Figure 1(e)). In this figure curves A, B C and D correspond to coatings with optical thicknesses adjusted to give 95, 55, 25 and 5\% transmittance at $560 \mathrm{~nm}$. The notional interrogation wavelengths used in these simulations were 560, 565, 575 and $590 \mathrm{~nm}$ respectively.

The other geometric extreme is provided by a sensing coating comprised of a single, relatively dense, layer of nanoparticles deposited on a substrate. We will designate this as the 'island geometry'. In this case the transmittance can be estimated from a literal interpretation of the definition of extinction cross-section:

$$
T \approx 1-\pi V a_{\text {eff }}^{2} Q_{\text {ext }}
$$

provided $\pi V a_{e f f}^{2} Q_{e x t}<1$. If $\pi V a_{e f f}^{2} Q_{\text {ext }}>1$ then $T \approx 0 . V$ is the density of particles per unit area.

Now

$$
S=\frac{\mathrm{d} T}{\mathrm{~d} n}=-\pi V a_{\text {eff }}^{2} \frac{\mathrm{d} Q_{e x t}}{\mathrm{~d} n}
$$

Expressions (5) and (7) produce similar estimates of $T$ when the samples are highly transparent, but deviate as the substrate becomes two-dimensional and less transparent. Furthermore, as the nanoparticles become more densely packed, particle-particle interactions occur that will red-shift the plasmon resonances [31]. We caution therefore that the expressions provided above are somewhat idealistic. They can be used to provide a first indication of which sensor designs will be the most sensitive, however, any actual sensor design should obviously be experimentally optimised and calibrated.

The key quantity that describes the performance of a monochromatic sensor is the change in transmittance, at the chosen wavelength, with respect to refractive index, evaluated at the nominal analyte refractive index. In the case of the nano-spheres, this quantity had a value of -252 at a medium refractive index of 1.40. Plasmon wavelength shift contributes to this parameter, but it is also influenced by peak sharpness. In general, a large shift combined with a sharp peak will result in high sensitivity, however, if this is too great then non-linearity and ultimately ambiguity can result. Thus it is important to operate near half-maximum cross- 
section. It can be shown that the performance for small refractive index changes can also be estimated using a linear model, as shown in Equation (9):

$$
\frac{d C}{d n} \sim \frac{d \lambda}{d n} \frac{1}{\Delta \lambda} C_{p}
$$

where $n$ is the index to be measured, and $C_{p}, \lambda_{p}$ and $\Delta \lambda$ are respectively the cross-section, centre wavelength and full-width-half-maximum of the peak. Some of these factors have been mentioned in relation to rods $[18,21]$, but here we explicitly examine the relative contributions for a variety of shapes. Although, strictly speaking, this model does not apply to more realistic refractive index changes due to the Lorentzian peak shape, it does provide an indication of the relative contributions to performance.

\section{Results and Discussion}

The optical responses from the nano-rods, nano-triangles and nano-bowties are given in Figures 2 to 6 , with the calculated efficiencies listed in Table 1.

Table 1 Simulated refractometric sensor results for the various shapes of nanoparticles.

\begin{tabular}{lllll}
\hline Shape & $\begin{array}{l}\text { Optimal } \\
\text { Wavelength }\end{array}$ & $\left(\frac{\mathrm{d}(\mathrm{T} \%)}{\mathrm{d} n}\right)$ & $\begin{array}{l}\text { Reference } \\
\text { transmittance } \\
\text { 'sweet-spot' }\end{array}$ & $\begin{array}{l}\text { Wavelength of } \\
\text { reference } \\
\text { transmittance }\end{array}$ \\
\hline $\begin{array}{llll}\text { nano-rods } \\
\text { (colloidal geometry) }\end{array}$ & $\begin{array}{l}1055 \mathrm{~nm} \\
1160 \mathrm{~nm}\end{array}$ & +665 & 10 to $15 \%$ & $1075 \mathrm{~nm}$ \\
$\begin{array}{l}\text { nano-rods on glass } \\
\text { (island geometry) }\end{array}$ & $970 \mathrm{~nm}$ & +1156 & 2 to $5 \%$ & $1075 \mathrm{~nm}$ \\
$\begin{array}{l}\text { nano-triangles } \\
\text { (colloidal geometry) }\end{array}$ & $7015 \mathrm{~nm}$ & -1162 & 25 to $35 \%$ & $1000 \mathrm{~nm}$ \\
$\begin{array}{l}\text { nano-triangles } \\
\text { glass }\end{array}$ & $730 \mathrm{~nm}$ & +350 & 65 to $75 \%$ & $700 \mathrm{~nm}$ \\
$\begin{array}{l}\text { (island geometry) } \\
\text { nano-bowtie on glass } \\
\text { (island geometry) }\end{array}$ & $720 \mathrm{~nm}$ & +609 & $65 \%$ & $710 \mathrm{~nm}$ \\
$\begin{array}{l}\text { nano-sphere } \\
\text { (colloidal geometry) }\end{array}$ & $575 \mathrm{~nm}$ & -591 & $5 \%$ & $750 \mathrm{~nm}$ \\
\hline
\end{tabular}




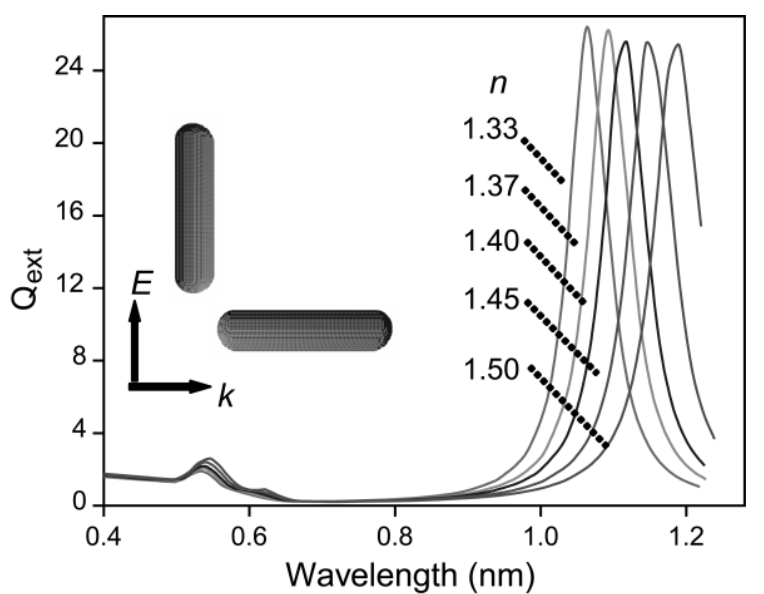

(a)

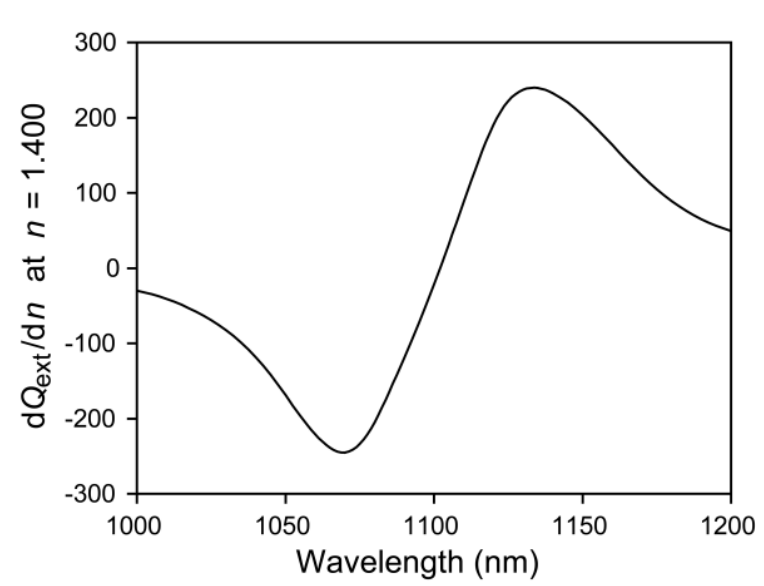

(b)

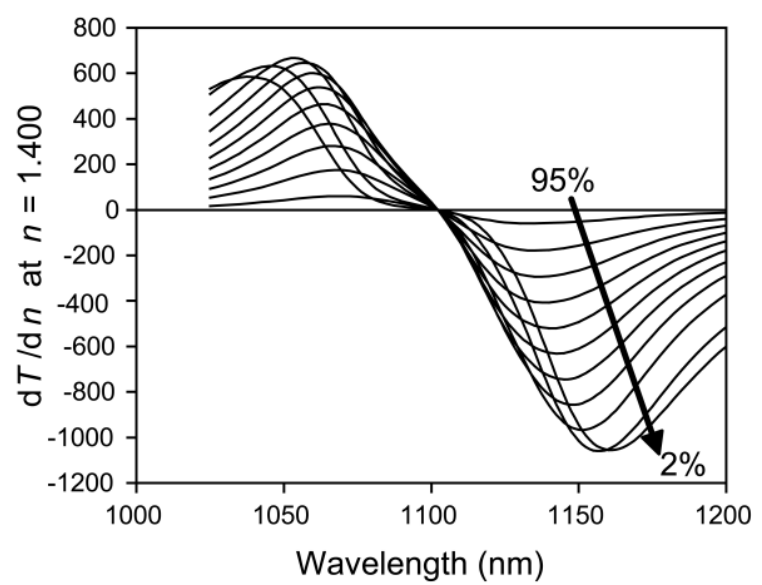

(c)

Figure 2 Optical response from nano-rods in colloidal suspension in media of varying refractive index. (a) Calculated extinction efficiency of nano-rods, (b) $\left|\frac{\mathrm{d} Q_{\text {ext }}}{\mathrm{d} n}\right|_{n=1.400}$ as a function of interrogation wavelength. (c) $\left(\frac{\mathrm{d}(\mathrm{T} \%)}{\mathrm{d} n}\right)_{n=1.400}$ as a function of wavelength, for coatings of the indicated optical thickness, parameterised as $\mathrm{T} \%$ at $1075 \mathrm{~nm}$.

Individual gold nano-rods in the colloidal geometry (Figure 2(a)) produce an intense optical response, with the narrowest peaks. The wavelength shift for the nano-rods is significant, about $700 \mathrm{~nm} \mathrm{RIU}{ }^{-1}$ in this somewhat idealised scenario and, given the sharpness of the peaks, it is expected that the nano-rods would be one of the more sensitive shapes for a single wavelength sensor. Calculation of $\left|\frac{\mathrm{d} Q_{e x t}}{\mathrm{~d} n}\right|_{n=1.400}$ shows that a monochromatic sensor could be interrogated on either side of the LSPR peak, Figure 2(b). Here we have evaluated $\mathrm{d} T / \mathrm{d} n$ at the optimum position on the right-hand (ie. red) side of the LSPR peak, Figure 2(c). Of course, practicalities in the 
application of a nano-rod sensor suggest a substrate will be required (eg. glass). Figure 3 shows the optical response of a layer of nano-rods on glass, applied in the island geometry. Here the overall extinction efficiency $\left(Q_{\text {ext }}\right)$ of the target has increased in comparison with the individual nano-rods in colloidal geometry, and there is also a reduced peak shift (about $500 \mathrm{~nm} \mathrm{RIU}{ }^{-1}$ ). More important than the peak-shift, however, is the overall sensor efficiency of the island geometries, which is exceedingly high, Table 1. The reason for this is the sharpness of the peaks for the nano-rod on glass, with a peak width of $\sim 33 \mathrm{~nm}$ for $n=1.33$ (at the full-width-halfmaximum), in comparison with $\sim 96 \mathrm{~nm}$ for $n=1.33$ for the individual nano-rods in colloidal suspension. This demonstrates that not only is the peak shift important, but the sharpness of the peak will also play an important part in overall sensor efficiency.

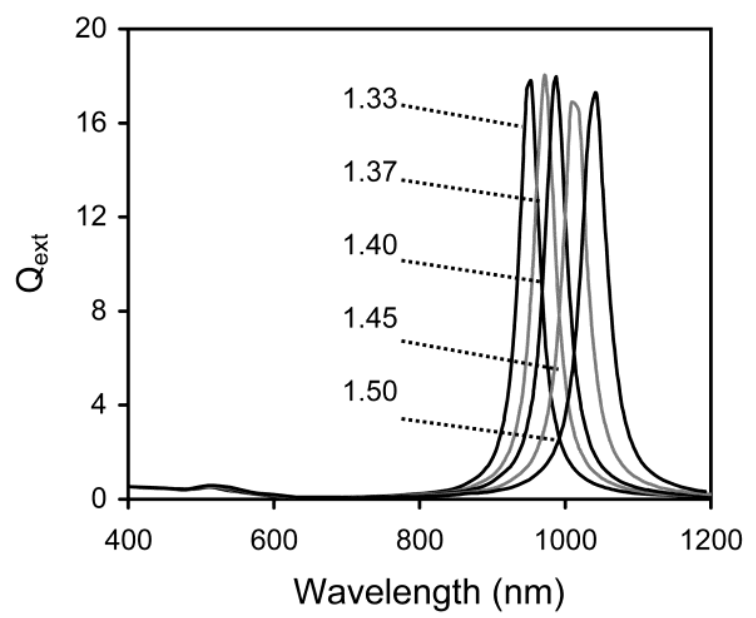

(a)

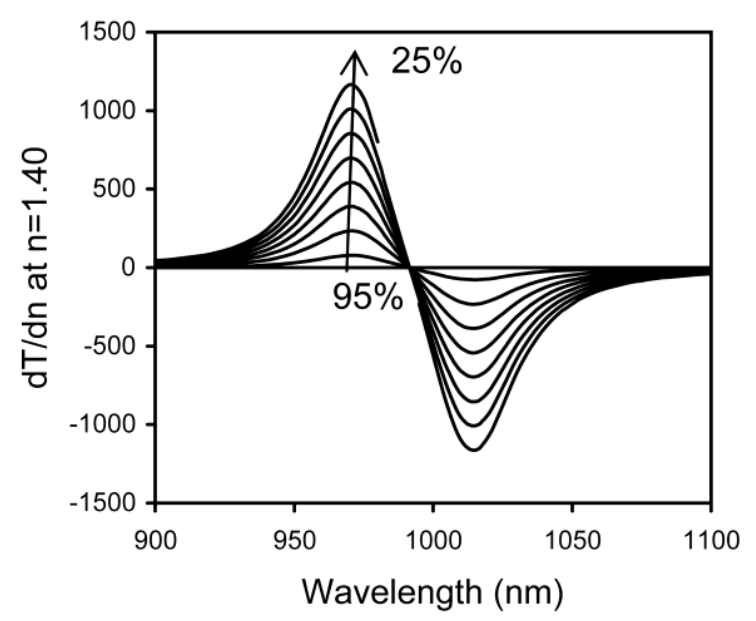

(b)

Figure 3 Optical response of coatings of $\mathrm{Au}$ nano-rods on glass. (a) Calculated extinction efficiency of nano-rods, (b) $\left(\frac{\mathrm{d}(\mathrm{T} \%)}{\mathrm{d} n}\right)_{n=1.400}$ as a function of wavelength, calculated for 'island geometry', for coatings of the indicated optical thickness, parameterised as $\mathrm{T} \%$ at $1000 \mathrm{~nm}$. There are two possible regions in which the sensor might be operated.

Nano-triangles (Figure 4) are significantly better than nano-spheres in terms of sensor efficiency with a centre-centre peak shift of $\sim 66 \mathrm{~nm}$, a relatively high $Q_{\text {ext }}$ value and reasonably sharp peak, as evaluated in the colloidal geometry. In this case there are also two regions of the spectrum where a sensor based on these particles could operate, corresponding to the left and right-hand sides of the LSPR peak. However, since $\left|\frac{\mathrm{d} Q_{\text {ext }}}{\mathrm{d} n}\right|_{n=1.400}$ was slighter larger $(\sim 78)$ at 735 $\mathrm{nm}$ on the left side than on the right hand side at $775 \mathrm{~nm}(\sim 68)$, we evaluated the operation on the left-hand side of the LSPR peak. Reasonable values of $\mathrm{d} T / \mathrm{d} n$ are obtained. 


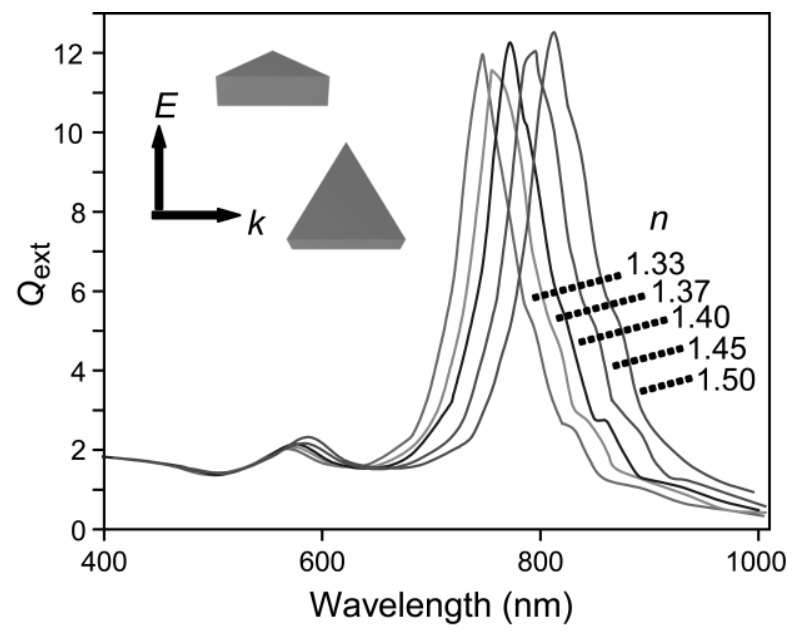

(a)

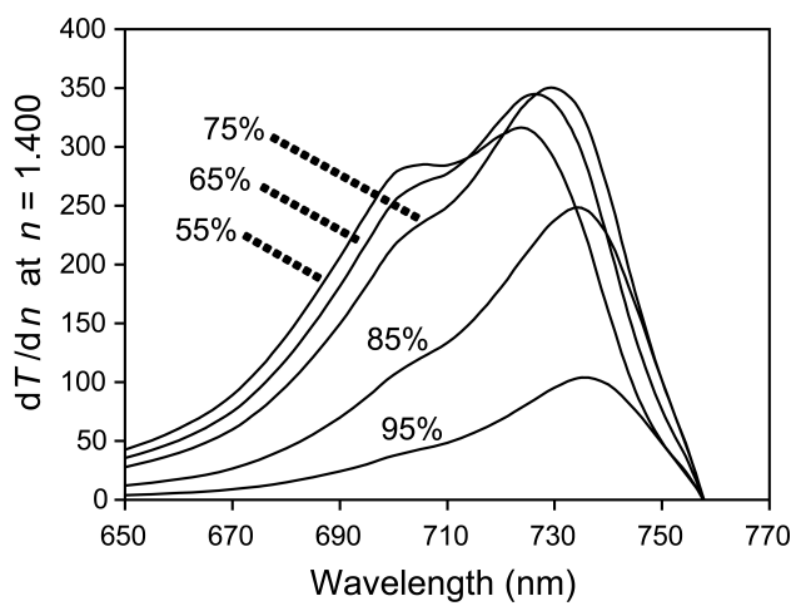

(b)

Figure 4 Optical response and refractometric sensitivity for gold nano-triangles in 'colloidal geometry'. (a) Extinction efficiency as a function of wavelength of light. (b) Sensitivity, $\left(\frac{\mathrm{d}(\mathrm{T} \%)}{\mathrm{d} n}\right)_{n=1.400}$, plotted for interrogation wavelengths in the range 650 to $750 \mathrm{~nm}$, for different optical thicknesses of coating, parameterised as transmittance at $700 \mathrm{~nm}$.

When the nano-triangle is placed on a substrate in the island geometry (eg. glass in Figure 5), there is a significant improvement in sensitivity but multipole resonances appear in the optical response, particularly on the right-hand (red) side of the LSPR. The multipole resonances would introduce noise into the signal, therefore we have evaluated the sensor response in this case for the left hand side of the LSPR peak. A shape combining two triangles, the nano-bowtie on glass (Figure 6), which must also be in also in the island geometry, provides similar sensitivity to the triangles on glass. 


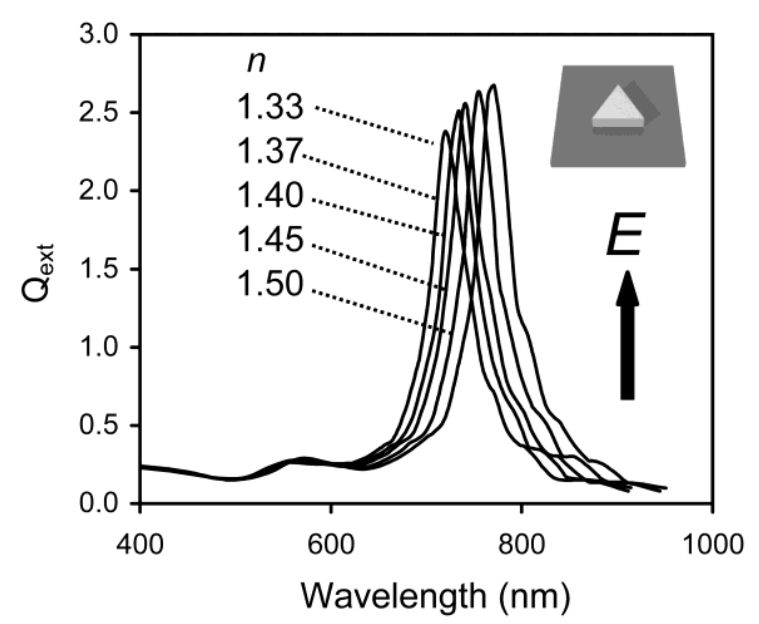

(a)

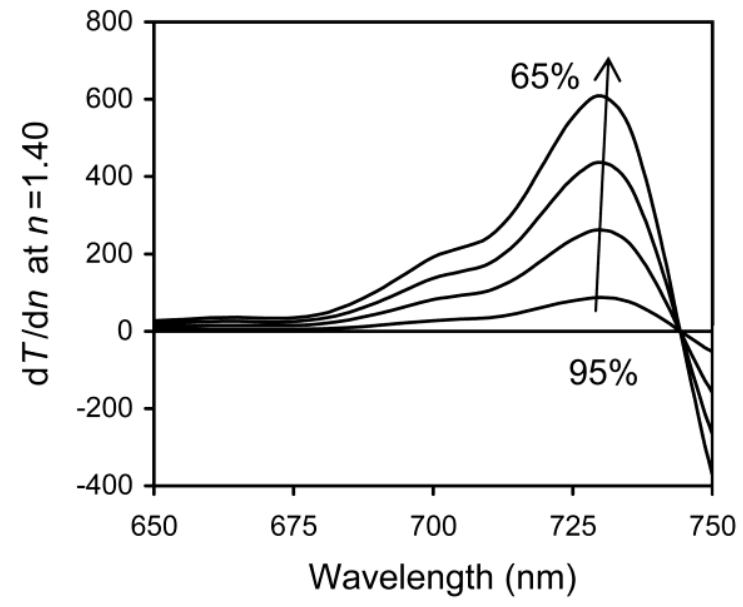

(b)

Figure 5 Optical response from a nano-triangle on glass, calculated using 'island geometry'. (a) Extinction efficiency as a function of wavelength of light. (b) Sensitivity, $\left(\frac{\mathrm{d}(\mathrm{T} \%)}{\mathrm{d} n}\right)_{n=1.400}$, plotted against interrogation wavelength, for different optical thicknesses of coating, parameterised as transmittance at $710 \mathrm{~nm}$.

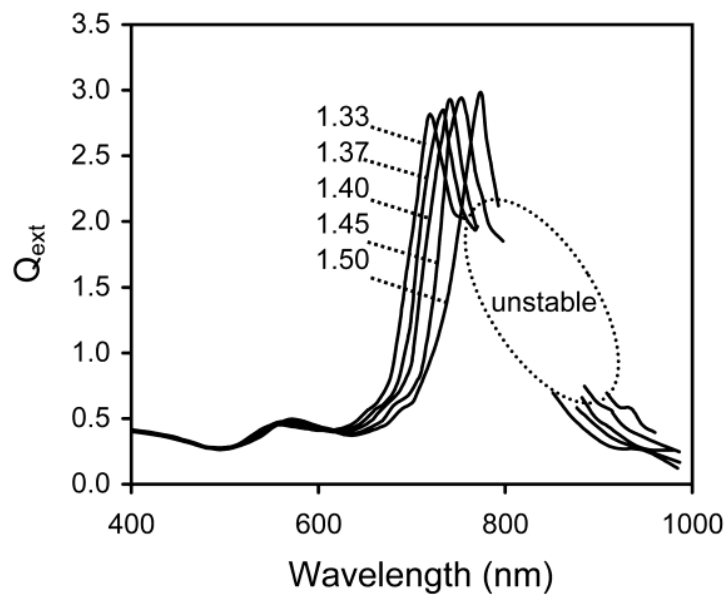

(a)

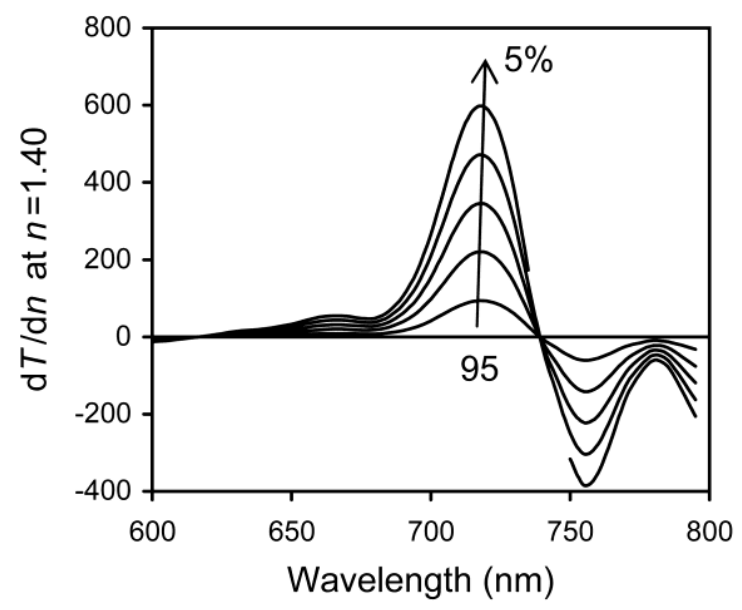

(b)

Figure 6 Optical response from a nano-bowtie on glass, calculated using 'island geometry'. (a) Extinction efficiency as a function of wavelength of light. The numerical technique was unstable in the region indicated (b) Sensitivity, $\left(\frac{\mathrm{d}(\mathrm{T} \%)}{\mathrm{d} n}\right)_{n=1.400}$, plotted against a range interrogation wavelengths for different optical thicknesses of coating, parameterised as transmittance at $750 \mathrm{~nm}$. 
As mentioned in the Methodology section, it is possible to calculate the performance for small refractive index changes using the linear model in Eq. (9). A summary of these performances, taking into account the dielectric characteristics of the gold (see Arnold and Blaber [32]) is given in Figure 7. Based on these performance indicators, it can be seen that the nano-rods produce the best over-all results, scoring highly for all four characteristics. Relatively, nano-rods have a high signal response, a significant wavelength shift, and a narrower plasmon peak in comparison with the other geometries trialled.

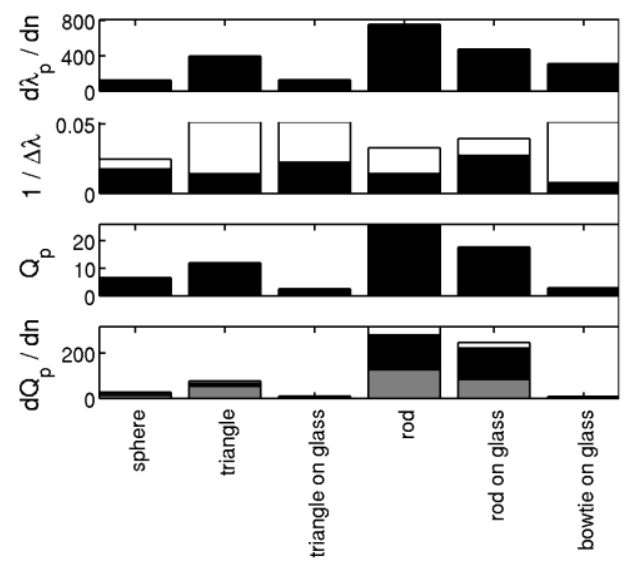

Figure 7 Summary of performance characteristics for the various geometries, averaged over refractive index, higher being better in every case. The upper three show the contributors to Equation (1) in black. The lower graph is a comparison of a complete linear model in white, Equation (1) in black, and the actual performance for the entire index range in gray. The secondary data for the inverse bandwidth, in white, is the potential result based on metal losses only. The units of wavelength are $\mathrm{nm}$.

\section{Conclusions}

The performance of a variety of different geometries of nanoparticles as the basis for single wavelength sensors of refractive index has been estimated. The results of this work show that gold nano-rods are the shape with the most potential for use in single wavelength refractometric sensor applications, based on both their plasmon wavelength shift and peak sharpness. The other shapes examined (nano-triangles, nano-bowties and nano-spheres) are far inferior in terms of the metrics defined and used here. Monochromatic refractometric sensors based on gold nano-rods seem viable and should be explored further. 


\section{References}

1. C.A. Berven and V.V. Dobrokhotov, Towards practicable sensors using one-dimensional nanostructures, Int. J. Nanotechnol., 5(4/5) (2008) 402-449.

2. C. Drake, S. Deshpande, D. Bera, and S. Seal, Metallic nanostructured materials based sensors, Int. Mater. Rev., 52(5) (2007) 289-317.

3. H. Haick, Chemical sensors based on molecularly modified metallic nanoparticles, J. Phys. D: Appl. Phys., 40 (2007) 7173-7186.

4. N. Stokes, A.M. McDonagh, and M.B. Cortie, Preparation of nanoscale gold structures by nanolithography, Gold Bull., 40 (2007) 310-320.

5. K.L. Kelly, E. Coronado, L.L. Zhao, and G.C. Schatz, The optical properties of metal nanoparticles: the influence of size, shape and dielectric environment, J. Phys. Chem. B, 107 (2003) 668-677.

6. E.C. Nice and B. Catimel, Instrumental biosensors: new perspectives for the analysis of biomolecular interactions, BioEssays, 21 (1999) 339-352.

7. T. Vo-Dinh, B. Cullum, and F. J., Biosensors and biochips: advances in biological and medical diagnostics, Anal. Chem., 366(6-7) (2000) 540-551.

8. S.A. Maier, Plasmonics. Fundamentals and Applications, Springer, New York, 2007.

9. H. Wang, D.W. Brandl, F. Lel, P. Nordlander, and N.J. Halas, Nanorice: a hybrid plasmonic nanostructure, Nano Lett., 6 (2006) 827-832.

10. A.J. Haes and R.P.V. Duyne, Preliminary studies and potential applications of localized surface plasmon resonance spectroscopy in medical diagnostics, Expert Rev. Mol. Diagn., 4(4) (2004) 527-537.

11. C. Drake, S. Deshpande, D. Bera, and S. Seal, Metallic nanostructured materials based sensors, International Materials Reviews, 52(5) (2007) 289-317.

12. C.R. Yonzon, D.A. Stuart, X. Zhang, A.D. McFarland, C.L. Haynes, and R.P. Van Duyne, Towards advanced chemical and biological nanosensors-An overview, Talanta, 67 (2005) 438-448.

13. C.R. Yonzon, E. Jeoung, S. Zou, G.C. Schatz, M. Mrksich, and R.P.V. Duyne, A comparative analysis of localized and propagating surface plasmon resonance sensors: the binding of concanavalin A to a monosaccharide functionalized self-assembled monolayer, Journal of the American Chemical Society, 126(39) (2004) 12669-12676.

14. G. Kalyuzhny, A. Vaskevich, A. Schneeweiss, and I. Rubinstein, Transmission surface plasmon resonance (T-SPR) measurements for monitoring adsorption on ultrathin gold island films, Chem. Eur. J. , 8 (2002) 3849-3857.

15. M.D. Malinsky, K.L. Kelly, G.C. Schatz, and R.P. Van Duyne, Chain length dependence and sensing capabilities of the localized surface plasmon resonance of silver nanoparticles chemically modified with alkanethiol self-assembled monolayers, J. Am. Chem. Soc. , 123 (2001) 1471-1482.

16. A.G. Brolo, R. Gordon, B. Leathem, and K.L. Kavanagh, Surface plasmon sensor based on the enhanced light transmission through arrays of nanoholes in gold films, Langmuir, 20(12) (2004) 4813-4815.

17. E. Hao, G.C. Schatz, and J.T. Hupp, Synthesis and optical properties of anisotropic metal nanoparticles, J. Fluorescence, 14(4) (2004) 331-341.

18. K.-S. Lee and M.A. El-Sayed, Gold and silver nanoparticles in sensing and imaging: sensitivity of plasmon response to size, shape, and metal composition, J. Phys. Chem. B, 110 (2006) 19220-19225. 
19. C.-D. Chen, S.-F. Cheng, L.-K. Chau, and C.R.C. Wang, Sensing capability of the localized surface plasmon resonance of gold nanorods, Biosens. Bioelectron., 22 (2007) 926-932.

20. H. Chen, X. Kou, Z. Yang, W. Ni, and J. Wang, Shape- and size-dependent refractive index sensitivity of gold nanoparticles, Langmuir, 24 (2008) 5233-5237.

21. G.J. Nusz, A.C. Curry, S.M. Marinakos, A. Wax, and A. Chilkoti, Rational selection of gold nanorod geometry for label-free plasmonic biosensors, ACS Nano, 3(4) (2009) 795806.

22. R. Bukasov and J.S. Shumaker-Parry, Highly tunable infrared extinction properties of gold nanocrescents, Nano Lett., 7 (2007) 1113-1118.

23. L.J. Sherry, S.H. Chang, G.C. Schatz, R.P. Van Duyne, B.J. Wiley, and Y.N. Xia, Localized surface plasmon resonance spectroscopy of single silver nanocubes, Nano Lett., 5 (2005) 2034-2038.

24. B.T. Draine and P.J. Flatau, Discrete-dipole approximation for scattering calculations, J. Opt. Soc. Am. A, 11 (1994) 1491-1499.

25. B.T. Draine and P.J. Flatau. User Guide to the Discrete Dipole Approximation Code DDSCAT 6.1. 2004 [cited January 2008]; Available from: http://arxiv.org/abs/astro$\mathrm{ph} / 0409262$.

26. A. Brioude, X.C. Jiang, and M.P. Pileni, Optical Properties of gold nanorods: DDA simulations supported by experiments, J. Phys. Chem. B, 109 (2005) 13138-13142.

27. N. Felidj, J. Aubard, and G. Levi, Discrete dipole approximation for ultraviolet-visible extinction spectra simulation of silver and gold colloids, J. Chem. Phys., 111 (1999) 1195-1208.

28. J.H. Weaver and H.P.R. Frederikse, in: D.R. Lide, (Ed.), CRC Press, Boca Raton, Florida, 2001.

29. N.L. Swanson and B.D. Billard, Optimization of extinction from surface plasmon resonances of gold nanoparticles, Nanotechnol., 14 (2003) 353-357.

30. J. Perez-Juste, I. Pastoriza-Santos, L.M. Liz-Marzan, and P. Mulvaney, Gold nanorods: synthesis, characterization and applications, Coord. Chem. Rev., 249 (2005) 1870-1901.

31. K.E. Peceros, X. Xu, S.R. Bulcock, and M.B. Cortie, Dipole-dipole plasmon interactions in gold-on-polystyrene composites, J. Phys. Chem. B, 109(46) (2005) 21516-21520.

32. M.D. Arnold and M.G. Blaber, Optical performance and metallic absorption in nanoplasmonic systems, Opt. Express 17(5) (2009) 3835-3847. 


\section{Biographies}

Catherine Kealley is a Post-Doctoral Researcher at the University of Technology Sydney. She holds a BSc(Hons) from Curtin University in Perth, Australia, and a PhD from University of Technology Sydney, Australia.

Matthew Arnold is a Lecturer at the University of Technology Sydney, Australia. He holds a $\mathrm{BSc}(\mathrm{Hons})$ and PhD in physics, both from the University of Otago in New Zealand.

Alexander Porkovich is a PhD Student at the University of Technology Sydney, Australia. He has a BSc(Hons) in Nanotechnology from the same University.

Michael Cortie has a $\mathrm{BSc}(\mathrm{Eng})$ and $\mathrm{PhD}$ in metallurgical engineering from the University of the

Witwatersrand, South Africa, and a M.Eng from the University of Pretoria in the same country. He is Director of the Institute for Nanoscale Technology at the University of Technology Sydney, Australia. 


\section{Figure captions}

Figure 1 Method used to simulate the efficiency of the sensor, using the example of a colloidal suspension of gold nano-spheres. (a) Calculated extinction efficiency, $Q_{\text {ext }}, v s$ wavelength, $\lambda$, for an individual $52 \mathrm{~nm}$ diameter gold sphere immersed in media of the indicated refractive index. (b) $Q_{\text {ext }}$ vs $n$ curves determined for interrogation wavelengths between 550 and $620 \mathrm{~nm}$. (c) $\mathrm{d} Q_{\text {ext }} / \mathrm{d} n$ evaluated at a nominal $n$ of 1.400 , and plotted against interrogation wavelength. (d) $\mathrm{d} T / \mathrm{d} n$ plotted against both wavelength and optical thickness. The reference transmittances shown are taken at $560 \mathrm{~nm}$. Curves A to D are representative examples. (e) Application of curves A to D in a monochromatic LSPR sensor using the concentration of glycerol in water as an illustrative example.

Figure 2 Optical response from nano-rods in colloidal suspension in media of varying refractive index. (a) Calculated extinction efficiency of nano-rods, (b) $\left|\frac{\mathrm{d} Q_{\text {ext }}}{\mathrm{d} n}\right|_{n=1.400}$ as a function of interrogation wavelength. (c) $\left(\frac{\mathrm{d}(\mathrm{T} \%)}{\mathrm{d} n}\right)_{n=1.400}$ as a function of wavelength, for coatings of the indicated optical thickness, parameterised as $\mathrm{T} \%$ at $1075 \mathrm{~nm}$.

Figure 3 Optical response of coatings of $\mathrm{Au}$ nano-rods on glass. (a) Calculated extinction efficiency of nano-rods, (b) $\left(\frac{\mathrm{d}(\mathrm{T} \%)}{\mathrm{d} n}\right)_{n=1.400}$ as a function of wavelength, calculated for 'island geometry', for coatings of the indicated optical thickness, parameterised as T\% at $1000 \mathrm{~nm}$. There are two possible regions in which the sensor might be operated.

Figure 4 Optical response and refractometric sensitivity for gold nano-triangles in 'colloidal geometry'. (a) Extinction efficiency as a function of wavelength of light. (b) Sensitivity, $\left(\frac{\mathrm{d}(\mathrm{T} \%)}{\mathrm{d} n}\right)_{n=1.400}$, plotted for interrogation wavelengths in the range 650 to $750 \mathrm{~nm}$, for different optical thicknesses of coating, parameterised as transmittance at $700 \mathrm{~nm}$.

Figure 5 Optical response from a nano-triangle on glass, calculated using 'island geometry'. (a) Extinction efficiency as a function of wavelength of light. (b) Sensitivity, $\left(\frac{\mathrm{d}(\mathrm{T} \%)}{\mathrm{d} n}\right)_{n=1.400}$, plotted 
against interrogation wavelength, for different optical thicknesses of coating, parameterised as transmittance at $710 \mathrm{~nm}$.

Figure 6 Optical response from a nano-bowtie on glass, calculated using 'island geometry'. (a) Extinction efficiency as a function of wavelength of light. The numerical technique was unstable in the region indicated (b) Sensitivity, $\left(\frac{\mathrm{d}(\mathrm{T} \%)}{\mathrm{d} n}\right)_{n=1.400}$, plotted against a range interrogation wavelengths for different optical thicknesses of coating, parameterised as transmittance at $750 \mathrm{~nm}$.

Figure 7 Summary of performance characteristics for the various geometries, averaged over refractive index, higher being better in every case. The upper three show the contributors to Equation (1) in black. The lower graph is a comparison of a complete linear model in white, Equation (1) in black, and the actual performance for the entire index range in gray. The secondary data for the inverse bandwidth, in white, is the potential result based on metal losses only. The units of wavelength are nm. 